\title{
The Current Epidemiology of Rotavirus Infection in Children Less than 5 Years of Age after Introduction of RV Vaccine in India
}

\author{
Ankur Kumar ${ }^{1}$ (D) Anita Pandey ${ }^{1 *}$ (D) Amresh Kumar Singh², \\ Archana Dubey ${ }^{1}$, Asmita Singh ${ }^{2}$ and Vivek Gaur ${ }^{2}$ \\ ${ }^{1}$ Department of Microbiology, Subharti Medical College, Swami Vivekanand Subharti University, \\ Meerut - 250 005, Uttar Pradesh, India. \\ ${ }^{2}$ Baba Raghav Das Medical College, Gorakhpur - 273 013, Uttar Pradesh, India.
}

\begin{abstract}
Rotavirus is a major cause of severe acute gastroenteritis in infants and young children worldwide. It is responsible for $40 \%$ of childhood hospitalization. It has been estimated that about $23 \%$ of diarrhoeal death occurs due to the rotavirus infection in India. To study the epidemiology of rotavirus infection in children less than $\mathbf{5}$ years of age at a tertiary care teaching hospital. A total of $\mathbf{2 4 0}$ stool samples were collected from children $<5$ years of age suffering from acute diarrhea at a tertiary health care referral hospital in Western Uttar Pradesh, India. Rotavirus antigen was detected in stool by Enzyme immunoassay (EIA) which utilizes monoclonal antibodies directed against VP6 antigen. Molecular genotyping was done by nested multiplex PCR. The rotavirus antigen positivity rate was found to be $14.58 \%$ in this study. There was male preponderance and the male: female ratio was 1.5:1. Rotavirus diarrhoea was reported predominantly $(42.85 \%)$ in the age group of 13 to 24 months. The most common circulating G/P genotype strain was G9P[4] accounting for $36 \%$ of cases. Rotavirus remains a major cause of diarrhoea in children $<5$ years of age in Uttar Pradesh. However, the positivity rate has decreased after introduction of rotavirus vaccine into the Universal immunization program (UIP) in UP.

Keywords: Diarrhoeal infection, Enzyme immunoassay (EIA), Rotavirus vaccine, rotavirus antigen
\end{abstract}

*Correspondence: anipanmicro@gmail.com

(Received: November 3, 2021; accepted: January 15, 2022)

Citation: Kumar A, Pandey A, Singh AK, Dubey A, Singh A, Gaur V. The Current Epidemiology of Rotavirus Infection in Children Less than 5 Years of Age after Introduction Of RV Vaccine in India. J Pure Appl Microbiol. 2022;16(1):471-480. doi: 10.22207/ JPAM.16.1.45

(C) The Author(s) 2022. Open Access. This article is distributed under the terms of the Creative Commons Attribution 4.0 International License which permits unrestricted use, sharing, distribution, and reproduction in any medium, provided you give appropriate credit to the original author(s) and the source, provide a link to the Creative Commons license, and indicate if changes were made. 


\section{INTRODUCTION}

Rotavirus still remains a leading cause of severe childhood diarrhea worldwide. World Health Organization (WHO) has estimated the occurrence of more than 25 million outpatient visits and more than 2 million hospitalizations attributable to rotavirus infections each year. ${ }^{1}$ Group A rotaviruses are responsible for severe acute gastroenteritis primarily in developing nations. ${ }^{2}$ In India, rotavirus is an important cause of viral gastroenteritis, affecting approximately 11.37 million children aged <5years annually and accounts for approximately $22 \%$ deaths associated with rotavirus gastroenteritis. ${ }^{3,4}$

Rotavirus represents a specific genus of the family Reoviridae, with a genome consisting of a double stranded RNA including 11 discrete segments or genes. Rotavirus possesses three concentric icosahedral protein shells of capsids. To date, on the basis of serological reactivity and amino acid sequence of protein VP6, ten distinct RV groups (or species) designated as A through J - have been identified in humans, other mammals and birds. Group (species) A RV is known to be the most common cause of diarrhoea in humans. ${ }^{1,5}$ Each RNA segment encodes for a single viral polypeptide including 6 structural (VP1, VP2, VP3, VP4, VP6, VP7): composing the core, inner shell, and outer shell; additionally, there are six nonstructural proteins including NSP1 to NSP6 play an important role in viral replication and growth. 5,6 Groups A-J can be differentiated with VP6 polyclonal and monoclonal antibodies by using immune fluorescence, Enzyme linked Immuno Sorbent Assay (ELISA) and immunoelectron Microscopy. ${ }^{6,7}$ The outer capsid proteins VP4 and VP7 are known to elicit type-specific neutralizing antibodies which may potentially confer protection against rotavirus infections caused by $\mathrm{G}$ types (VP7 protein) and $\mathrm{P}$ types (VP4 protein). To date $32 \mathrm{G}$ types and $42 \mathrm{P}$ types of Species $\mathrm{A}$ rotavirus have been identified although globally, six $G$ types (that is, G1, G2, G3, G4, G9 and G12) and three P types (that is, $P[4], P[6]$ and $P[8]$ ) predominate. ${ }^{5}$

Rotavirus is primarily transmitted by faecal-oral route directly from person to person and indirectly through the consumption of virus contaminated food and water. However, it can be transmitted by other methods like contaminated environmental surfaces, fomites and less commonly respiratory route. ${ }^{5,7,8} \mathrm{RV}$ infection produces a wide spectrum of clinical presentation ranging from asymptomatic/subclinical infection to severe, life-threatening illness with diarrhoea, vomiting and fever. Incubation period is short 24 to 72 hours and symptoms usually resolve in about three to seven days but shedding of rotavirus may persist for longer period in immune-compromised children. ${ }^{9,10}$

The impact of RV vaccination drastically reduced the morbidity and mortality in children worldwide. Recently published studies have shown that after introduction of vaccines in 43 countries in their study region trends avert the 710000 rotavirus hospitalizations and 35000 rotavirus deaths annually. Data published in studies in south East Asia showed that vaccine effectiveness ranges between 49 and $86 \%$ and vaccines were highly effective against severe RV infection during childhood. ${ }^{11}$

Availability of effective vaccine has warranted in controlling and preventing the infection as proved in previous epidemiological studies. ${ }^{11-13}$ There are two commercially available vaccine Rotarix (GlaxoSmithKline Biologicals SA, Belgium) RV1; monovalent vaccine containing G1P[8] strain and RotaTeq (Merck \& Co., Inc., USA) RV5; pentavalent containing G1-G4 P[8] have been available since 2006 in India. In 2015, the ROTAVAC vaccine (Bharat Biotech, India), containing the live $116 \mathrm{E}$ rotavirus strain (G9P [11]), was introduced as the first indigenously developed vaccine. i.e. In April 2016, the vaccine was introduced into the UIP by the Government of India. Another pentavalent vaccine (G1-G4, G9) ROTASIIL was prequalified in 2018 (Serum Institute of India Pvt. Ltd., Pune, India). However, the ROTASIIL vaccine is currently only in use in India. Rotavirus vaccines have demonstrated impact in reducing diarrheal morbidity and mortality worldwide. ${ }^{11-14}$

In Indian scenario, there is limited data available to estimate the burden of RV infection and status of RV associated morbidity and mortality in young children after introduction of vaccine; therefore we need to conduct nationwide surveillance studies to understand the epidemiology of rotavirus post vaccine introduction in India. ${ }^{12-14}$ Therefore our study was carried out to determine the positivity rates and the epidemiological features of rotavirus 
infection in children $<5$ years of age from Meerut district post-introduction of rotavirus vaccine.

\section{MATERIALS AND METHODS}

This cross sectional descriptive study was conducted at a tertiary care hospital, over the period of 2 years, from December 2018 to January 2020. Stool samples were collected from clinically suspected children aged less than 5 years. A total of 240 diarrheal cases were enrolled fulfilling study criteria which targeted children $<5$ years of age presenting with loose, watery stool. Children with dysentery (diarrhoea containing blood or mucus) and those with loose stool lasting $>14$ days and children who developed acute onset of loose stools $\geq 48$ hours after hospitalisation were excluded from the present study. The relevant clinical information was obtained from consulting the paediatrician and informed consent and requisition form was taken from parents or guardians at the time of sample collection in pre designed proforma as per WHO guidelines. ${ }^{15,16}$ The study was ethically approved by Institutional Review Board before enrolling the children.

Sample collection, transportation: Stool samples were collected in clean sterile container from children within 48 hours of illness and processed for rotavirus antigen detection by Enzyme Immuno- Assay (EIA) in the serology laboratory, department of microbiology. The clinical severity of rotavirus associated diarrhoea was recorded by using the 20-point Vesikari Clinical Severity Scoring System. ${ }^{17}$

\section{Sample processing}

In the laboratory, all stool samples were subjected to rotavirus antigen detection using commercial EIA (Premier Rotaclone meridian Bioscience Catalog: 696004, USA) as per manufacturers' instructions. Rotavirus detection and characterization were performed as per WHO protocol. ${ }^{15}$ This is a sandwich-type commercial assay that utilizes monoclonal antibodies directed at the conserved VP6 protein which is a groupspecific antigen for all known species $A$ human rotaviruses. The RV antigen concentration in sample is directly proportional the intensity of blue color. Two drops of stop solution were added in each well to stop the reaction and yellow color developed. The optical density was further measured by an ELISA reader, using different wavelength through primary and secondary filters at $450 \mathrm{~nm}$ followed by $620 \mathrm{~nm}^{15}$

\section{Molecular genotyping}

All EIA rotavirus-positive samples were further tested in National Rotavirus Surveillance laboratory, CMC Vellore Tamil Nadu for genotyping of rotavirus. Genotyping of RV was done by nested multiplex PCR by using homologous primer sequences as per WHO laboratory protocol. ${ }^{15}$

The data was summarized into tables, graphs, charts and proportions/percentages by using Microsoft Excel (Microsoft Office 2010 Professional). The $95 \%$ confidence interval, mean, standard deviation, and $p$ values were calculated to analyse the statistical significance of the study.

\section{RESULTS}

\section{Demographic distribution of Rotavirus}

Overall rotavirus antigen positivity rate in children $<5$ years of age with acute diarrhoea from Meerut district was $35(14.58 \%)$ Fig. 1 . The positivity rate was high in $13-24$ months ( $<2$ years) followed by in 7-12 months ( $<1$ year). Lowest positivity rate was seen in $0-6$ months [Table 1 ]. There was male predominance $(60 \%)$ and the male: female ratio was 1.5:1. (Fig. 2)

Table 1. Age distribution of rotavirus positive cases $(n=240)$

\begin{tabular}{|c|c|c|c|c|c|}
\hline Age (Months) & $\begin{array}{l}\text { Total tested } \\
(n=240)\end{array}$ & Male & Female & $\begin{array}{c}\text { Total } \\
\text { positive }\end{array}$ & $\begin{array}{c}\text { Percentage } \\
(\%)\end{array}$ \\
\hline 0 - 6 Months & 48 & 02 & 01 & 03 & 6.25 \\
\hline 7 - 12 Months & 71 & 04 & 07 & 11 & 15.49 \\
\hline $13-24$ Months & 66 & 11 & 04 & 15 & 22.72 \\
\hline 25 - 60 Months & 55 & 04 & 02 & 06 & 10.90 \\
\hline Total & 240 & 21 & 14 & 35 & 14.58 \\
\hline
\end{tabular}




\section{Clinical profile of Rotavirus positive cases}

According to the Ruuska and Vesikari scoring system, severity scores above 10 points (i.e., $\geq 11$ points) are considered as severe, scores between 7 and 10 are considered as moderate, and scores less than 7 are considered as mild. In this study average score lied between 7 to 10 points hence the majority of children 19 (54.2\%) were moderately ill followed by $10(28.5 \%)$ children severely ill and 6 (17.1\%) children were reported as having mild illness, as shown in Table 2. Among the rotavirus-positive cases, fever was reported in $(82.85 \%)$ of cases, being the most common clinical symptom. Overall, 28 (80\%) out of 35 rotaviruspositive children developed dehydration, including severe $(9 / 35 ; 25.71 \%)$, moderate $(17 / 35 ; 48.57 \%)$, and mild $(2 / 35 ; 5.71 \%)$ dehydration cases. Other important clinical signs including vomiting
(65.71\%), and loose stools with $\geq 6$ episodes/day $(62.8 \%)$ were also detected frequently, as shown in Table 3.

Role of risk factors in rotavirus infection in children

Risk assessment among rotavirus diarrheal cases is shown in Table 4. The majority of cases were enrolled at the inpatient department (IPD) ( $n=159)$ and only 81 patients had visited the outpatient department (OPD). Out of 35 RV-positive cases (both urban and rural areas included), 10 (28.5\%) OPD cases and 25 (71.4\%) IPD cases were found. It reflects the fact that the need for hospitalization in RV positive cases can be regarded as an essential indicator for assessing the economic burden of RV infection in developing nations. Of the 35 RV-positive cases, 17 (48.5\%) cases were from the rural area and $18(51.4)$ cases

\section{Rotavirus positivity among children below the age 5 years}

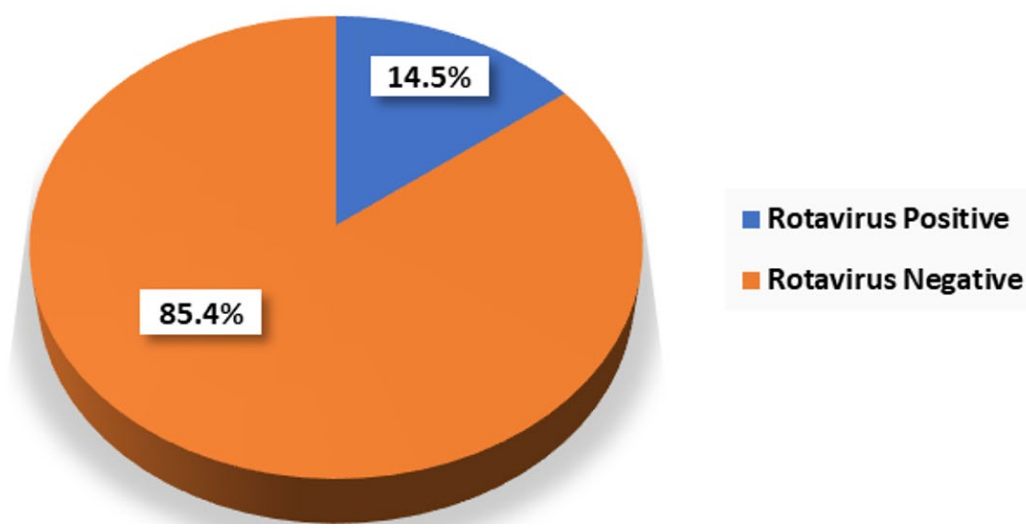

Fig. 1. Rotavirus positivity rate by EIA in children $<5$ years of age $(n=240)$.

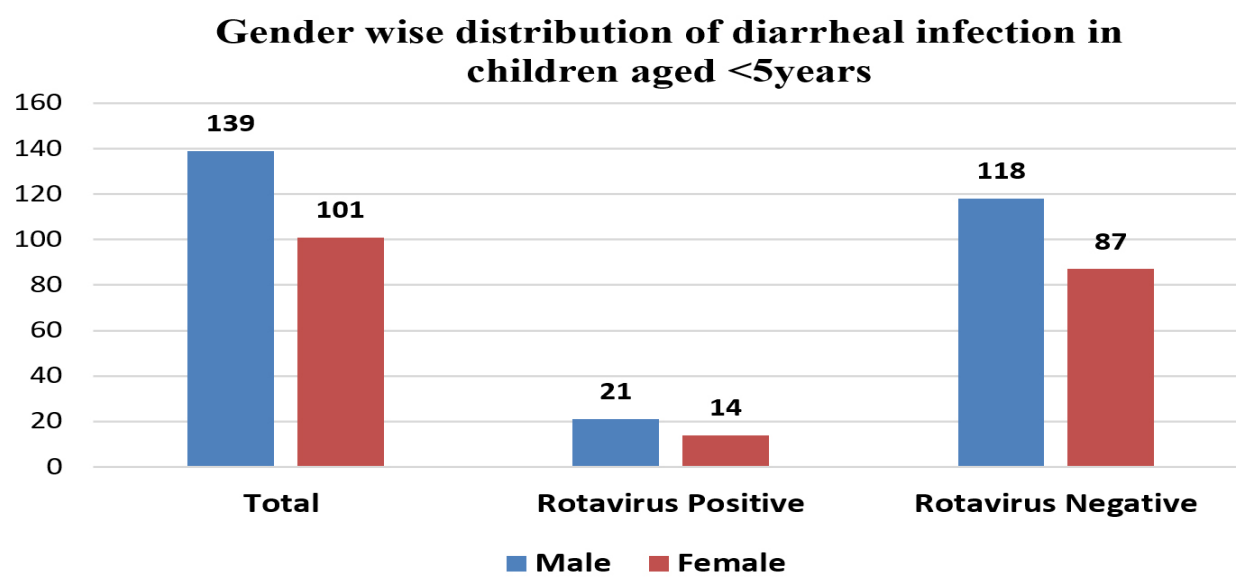

Fig. 2. Gender wise distribution of children $<5$ years. 
were from the urban area. Positivity rate was slightly higher in the urban population $(51.42 \%)$ as compared to the rural population (48.57\%) but statistically no significant difference was observed among RV-positive cases.

In this study, we considered malnutrition as one of the relevant risk factors among rotavirusassociated diarrheal children, as claimed in a previous study; thus we ruled out all the study samples to find out the co-relation of diarrhea and malnutrition. Malnutrition was seen in 76/240 cases of diarrhea and out of the malnourished children $14 / 35$ (40\%) cases were rotaviruspositive, and among these, 5 children were from the urban area and 9 children were from rural area (Table 4).

The literacy status of the mother plays an important role in awareness and hygiene practice of child as a preventive measure against diarrheal disease. In this study, 162/240 (67.5\%) mothers were literate (as defined by the Census of India under Ministry of Home affairs, Govt. of India). Taking the 35 RV-positive children together, 15 $(42.8 \%)$ of their mothers were literate, whereas

Table 2. Severity scoring as per Vesikari clinical severity scoring system

\begin{tabular}{lccc}
\hline $\begin{array}{l}\text { severity } \\
\text { scoring }\end{array}$ & $\begin{array}{c}\text { Total no. of } \\
\text { cases } \\
(\mathrm{n}=240)\end{array}$ & $\begin{array}{c}\text { RV Positive } \\
\text { cases } \\
(\mathrm{n}=35)\end{array}$ & $\begin{array}{c}\text { Percentage } \\
(\%)\end{array}$ \\
\hline Mild & 105 & 06 & $28.5 \%)$ \\
Moderate & 86 & 19 & $54.2 \%$ \\
Severe & 49 & 10 & $17.1 \%$ \\
\hline
\end{tabular}

20 (57.2\%) mothers were illiterate as shown in Table 4.

The feeding pattern of children represents one of the relevant risk factors which might be considered when assessing the risk for developing diarrheal disease. In this study, the majority of children were either bottle-fed or receiving replacement feeding $17 / 35$ (48.5\%), whereas $12 / 35(34.3 \%)$ children were on both breastfeeding and bottle-fed feeding. Only $6 / 35$ (17.2\%) children were being exclusively breast-fed.

The infections were comparatively higher in winters (October to February) than in summer months (March to June). The lowest number of cases were reported during the monsoon months (rainy season), from July to September (Fig. 3)

A total of 154/240 (64\%) children enrolled in the study were vaccinated with Rotavac (Bharat

Table 3. Clinical profile of children with diarrhoea $(n=240)$

\begin{tabular}{lcc}
\hline Clinical profile & $\begin{array}{c}\text { No. of } \\
\text { cases } \\
\mathrm{n}=240\end{array}$ & $\begin{array}{c}\text { Rotavirus } \\
\text { Positive } \\
\mathrm{n}=35(\%)\end{array}$ \\
\hline I Diarrhoea & 238 & $35(100 \%)$ \\
1- 3 Episodes/day & 51 & $04(11.42 \%)$ \\
4- 5 Episodes/day & 65 & $09(25.71 \%)$ \\
$\geq 6$ Episodes/day & 122 & $22(62.85 \%)$ \\
II Vomiting & 147 & $23(65.71 \%)$ \\
III Dehydration & 194 & $28(80 \%)$ \\
(a) Severe & 76 & $09(25.71 \%)$ \\
(b) Moderate & 108 & $17(48.57 \%)$ \\
(c) Mild & 10 & $02(5.71 \%)$ \\
IV Fever & 172 & $29(82.85 \%)$ \\
\hline
\end{tabular}

Seasonal variation of rotavirus positive and negative diarrhea children

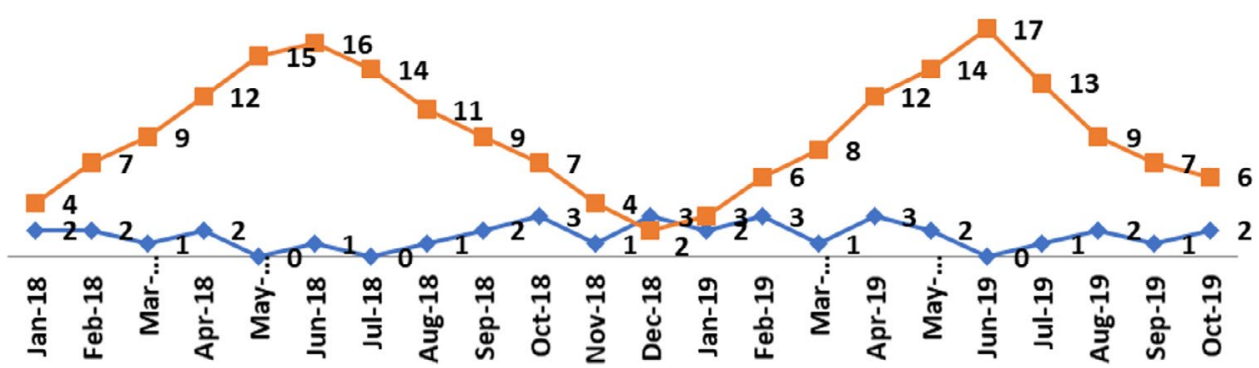

Fig. 3. Seasonal variation of rotavirus positive and negative cases $(n=240)$. 
biotech, India) introduced under UIP by Govt. of India, Out of 154 vaccinated children, 61\% were from urban areas and $38 \%$ belonged to a rural population. (Fig. 4). In this study, 86 (35.8\%) children did not receive any rotavirus vaccine dose. The rotavirus antigen positivity was seen in only $4 / 35$ children who had been vaccinated but in none of them rotavirus vaccine strains were detected.

\section{Genotypic distribution of Rotavirus}

G9 was the commonest (36\%) G genotype identified followed by G3 (26\%), G2 (13\%), and G1 and G12 (10\% each). P [4] type was the most common $\mathrm{P}$ genotype reported (53\%) followed by P [8] (40\%) and P [6] (10\%). Mixed type P [4 + 8] was also reported in 2 cases (6\%) (Fig. 5).

In this geographical area, the dual G9P[4] G/P combination was the predominant (36\%) genotype followed by G3P [8] (26\%) and G1P [8] (10\%). G2P [4], G2P [6] and G2P [8] were other less common genotypes identified [Fig. 5]. Uncommon strains like G12P [6] and G12P [8] were also reported in $3 \%$ of cases, as circulating genotypes in this area.

\section{DISCUSSION}

The positivity rate of rotavirus in this study was $14.58 \%$. This is slightly lower as compared to other studies reported from India by John et al and Banerjee et al. $24 \%$ and $27.4 \%$ respectively. ${ }^{18,19}$ The

Table 4. Risk assessment of RV positive cases with diarrheal disease $(n=35)$

\begin{tabular}{lcccc}
\hline \multirow{2}{*}{ Parameters } & $\begin{array}{c}\text { Total cases } \\
\mathrm{n}=240\end{array}$ & \multicolumn{2}{c}{$\begin{array}{c}\text { Rotavirus Positive } \\
(\mathrm{n}=35)\end{array}$} & Total (\%) \\
\cline { 3 - 4 } & & $\begin{array}{c}\text { Urban } \\
(\mathrm{n}=18)\end{array}$ & $\begin{array}{c}\text { Rural } \\
(\mathrm{n}=17)\end{array}$ \\
\hline OPD cases & & 07 & 03 & $10(28.5 \%)$ \\
IPD cases & 81 & 09 & 16 & $25(71.4 \%)$ \\
Malnutrition & 159 & 09 & 09 & $14(40 \%)$ \\
Mother literacy $\quad$ Literate & 162 & 05 & 10 & $15(42.8 \%)$ \\
(n=234) & 72 & 06 & 14 & $20(57.2 \%)$ \\
Breast feeding only & 43 & 02 & 04 & $6(17.2 \%)$ \\
Replacement (top) feeding & 110 & 11 & 06 & $17(48.5 \%)$ \\
Breast + Top feeding & 87 & 03 & 09 & $12(34.3 \%)$ \\
Vaccination status & 154 & 01 & 03 & $4(11.4 \%)$ \\
\hline
\end{tabular}

\section{RV vaccination status}

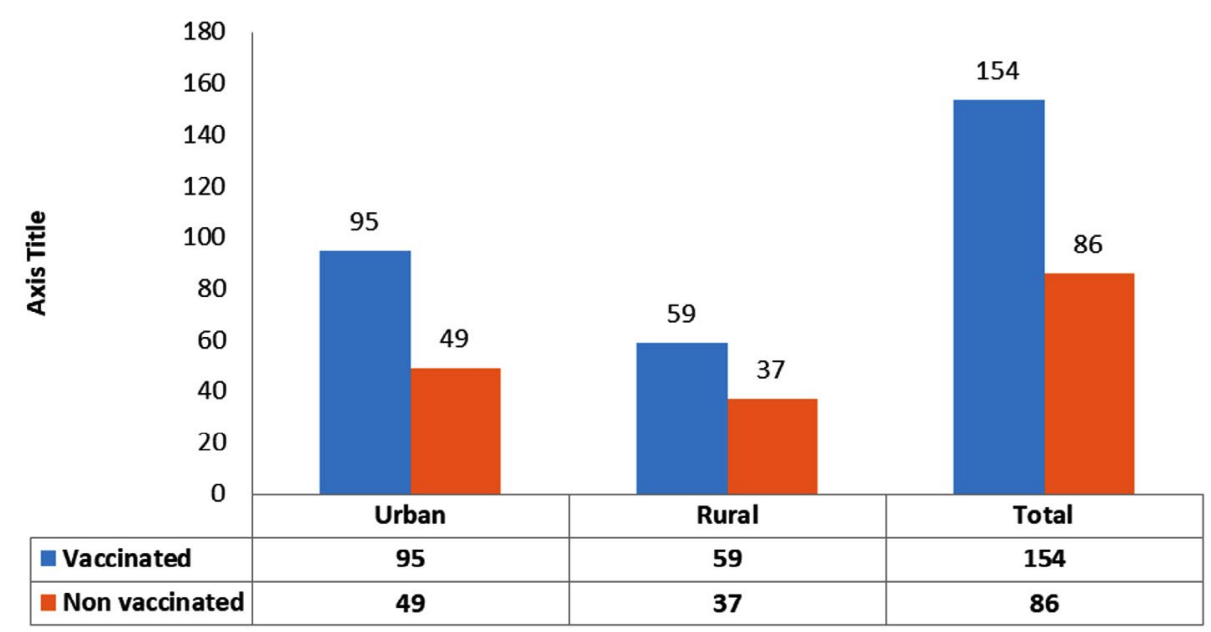

Fig. 4. Rotavirus vaccination status among the urban and rural population $(n=240)$. 
lower rate of rotavirus infection in this study may be due to the large-scale vaccination coverage under Universal immunization Programme or lack of enough rotavirus studies post-vaccination in India.

Our study showed male predominance (60\%), suggesting that male children were more prone to rotavirus infection than female. Similar finding has also been reported by Chavan et al. 20. The rotavirus infection was predominantly seen in $<2$ years of age $(42.85 \%)$ and was lowest $(17.14 \%)$ in children $<6$ months of age, which correlates well with several studies conducted in India. ${ }^{21,22}$ However, Dutta et al. showed that rotavirus infection was the most common (26.6\%) in children aged 6-11 months. ${ }^{23}$ The infections were predominantly (50\%) seen in the winter months (October to February), followed by $(31 \%)$ in summer months (March to June; $31 \%$ ). Lowest rate of infections (20\%) was seen during monsoon months. Our findings correlate well with other studies conducted in India in the past. ${ }^{14,24-}$ 26 This may be due to the fact that rotavirus survives enormously in cooler months and it has been hypothesized that spreading of rotavirus in cooler conditions occurs relatively in low humidity and infrequent rainfall in winter months, combined with drying of soils. Altogether these conditions might account for increasing spread of contaminated faecal material, as also reported in previous studies. ${ }^{14,25,26}$

In the present study the most common clinical symptoms of rotavirus infections was fever, vomiting, frequent $(>6)$ diarrhoeal episodes and dehydration. As per Ruuska \& Vesikari scoring system the majority of cases were classified as moderate. ${ }^{4,26-28}$

The majority of rotavirus-positive cases, $(71.42 \%)$ were IPD patients and (28.58\%) cases were OPD patients. This finding correlates well with other studies. ${ }^{10,18}$ Severe dehydration was the main clinical sign which has required hospitalization. In the present study most of the cases were found to be either clinically moderate or severe based on criteria stated by the Ruuska $\&$ Vesikari scoring system. Noticeably, no deaths have been as outcome of these severe episodes.

A total of $64.17 \%$ of children were vaccinated against rotavirus in our study population. Of note, a significant increase in rotavirus vaccine coverage has been seen since the introduction of Rotavac ${ }^{\circledR}$ indigenous, live oral vaccine (Bharat biotech, India). Rotavac ${ }^{\circledR}$ vaccine was introduced in 2016 in universal Immunization program of India and it was introduced in Uttar Pradesh since 2018 under UIP. Rotavirus vaccination coverage was relatively high as compared to coverage rates of only $3.5 \%$

\section{Distribution of Rotavirus Genotypes}

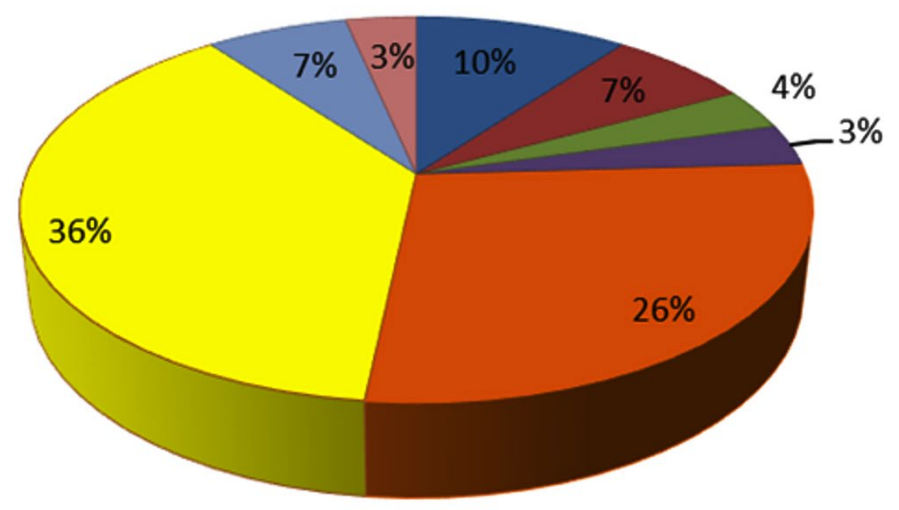

$\square \mathrm{G} 1 \mathrm{P}[8]$

口G2 P[4]

$\square \mathrm{G} 2 \mathrm{P}[6]$

G2P [8]

G3 P[8]

口G9P[4]

$\square \mathrm{G} 12 \mathrm{P}[6]$

$\square \mathrm{G12P}[8]$

Fig. 5. Genotypic distribution of Rotavirus strains in children. 
reported in a recent surveillance study conducted by Kumar et al. ${ }^{26}$ Our study also revealed that the $\mathrm{RV}$ vaccination coverage in children was quite higher in the study area as compared to studies conducted in other settings. ${ }^{25}$ These findings support the notion that effective implementation of universal Rotavirus vaccination significantly reduce the rates of rotavirus infection. ${ }^{27}$

In our study, the majority children ( $n=95)$ were from urban areas as compared to rural areas, highlighting the fact that rotavirus vaccination awareness \& surveillance program are needed to be conducted more frequently and effectively in the healhcare settings, in order to improve country-wide vaccination coverage. ${ }^{27}$

With regards to the assessment of risk factors for rotavirus infection, as feeding type, it was seen that most of the infections (45.83\%) have occurred in children who were either exclusively on breast-feeding or in children who were on both top (replacement) feeding and breast feeding. Rotavirus infection rate was lowest (17.91\%) in children who were exclusively on breast feeding, as compared to those who were bottle-fed. Similar observations of increased rotavirus infections among bottle-fed children were reported elsewhere. ${ }^{18}$ Nevertheless, poor hygiene practices among the top fed children may also have accounted for the higher rotavirus transmission among them.

Education of the parents, especially the mother, is a major factor affecting rotavirus diarrhoea, as these viruses are transmitted commonly by faecal-oral route. It was observed that children of less or illiterate mothers were more prone to infection, as compared to literate mothers. Several studies have also proved that education of mother plays an important role among measures towards prevention of rotavirus infection in young children, besides hygiene and sanitization awareness and active participation in child health program such as Navjaat Shishu Suraksha Karyakram (NSSK). 8,25,26

Malnutrition was seen in (18.42\%) of children in the present study. Either undernutrition or malnutrition has been reported as one important factor to reduce the risk of severe diarrhea in children. In this study, however, no significant correlation was observed in any of the age groups, unlike what has been reported earlier. ${ }^{28,29}$ Muendo et al. claimed that, hypothetically, malnutrition might be associated with protection due to various reasons, for instance, shortening of villi in malnourished children, hence leading to lesser absorption. ${ }^{29}$

In this study, the most common rotavirus G/P genotype was found to be G9P[4], followed by G3P[8] and G1P[8]. We also found that G12P[6] and G12P[8] were uncommon emerging strains in our study. Moreover, other studies conducted by Shrivastava et al. ${ }^{8}$ reported the most common strains as being G3P[8], followed by G1P(8), G2P[P4], G1P[P6], and G3P[P6]. Another study by Mathew et al. ${ }^{30}$ showed that G1P8 was the most common strain identified followed by G9P[8] G2P[4]. The circulating strains identified in our study, as compared to other studies, highlights the changing pattern of strains resulting in broad genomic diversity of rotavirus distribution in different geographical areas across the country. Babji et al. ${ }^{31}$ highlighted that G10P[11] was the most common and widespread strain in Vellore and Chennai, and owing to its high transmissibility, this specific neonatal strain continue to persist circulate over a decade. Few recent studies have quite different and diverse genotypic rotavirus strains distribution, which may vary due to sample size, study period, and geographical variation in different settings. ${ }^{8,10,28,31}$ However, the increasing trends and emergence of G9P[4] strains was observed throughout the study; it is therefore imperative to conduct regular surveillance to continuously monitor the trends of circulating RV strains to assess the impact of rotavirus vaccination on strain distribution and diversity. ${ }^{26}$

\section{CONCLUSION}

This study shows a decrease in the prevalence rate of rotavirus diarrhoea after intervention with rotavirus vaccination under the UIP in this region. Rotavirus diarrhoea is a vaccine-preventable disease; therefore, safe water, hygiene practice, awareness, and vaccine coverage are the key to stop further spreading of infection. Additional large scale, evidence-based studies from different geographical regions of India are required to accurately estimate the high burden of disease, genomic viral diversity, effectiveness of vaccine. In this study, G9P[4] was the most common RV strain reported (48\%), followed by 
G3P[8] was the reported in (17\% ) cases. Several other study showed that reassortment events and genetic mutations were found to be responsible for the rise of G9P [4] and strains G12P[6] and G12P[8] strains in the developing nations. Identification of circulating rotavirus strains and effective administration of vaccine play an important role toward the control and prevention of rotavirus infection.

\section{ACKNOWLEDGMENTS}

The authors would like to thank Dr. Gagandeep Kang \& Dr. Tintu Verghee, the Wellcome Trust Research Laboratory, Division of Gastrointestinal Sciences, Christian Medical College, Vellore, TN, India for providing support in the Genotypic Characterization of Rotavirus. Viral diagnostic and Research laboratory (VDRL), Department of Microbiology, BRD Medical College, Gorakhpur, UP, India under state government for assistance on methods and research. The authors would also like to thank all the laboratory technicians for their technical support in the completion of this study.

\section{CONFLICT OF INTEREST}

The authors declare that there is no conflict of interest.

\section{FUNDING}

None.

\section{AUTHORS' CONTRIBUTION}

All the authors listed have made a substantial, direct and intellectual contribution to the work, and approved it for publication.

\section{DATA AVAILABILITY}

All datasets generated or analyzed during this study are included in the manuscript and genetic characterization of microorganism their primers information was extracted from World Health Organization literature and NCBI gene bank USA.

\section{ETHICS STATEMENT}

The study was approved by the Institutional Ethical and Research committee Swami Vivekanand Subharti University, Uttar Pradesh, India (No. SMC/EC/2017/133)

\section{REFERENCES}

1. Rotavirus Epidemiology. World Health Organization (WHO) Global Report factsheet. https://www.who. int/teams/health-product-policy-and-standards/ standards-and-specifications/vaccines-quality/ rotavirus

2. Parashar UD, Hummelman EG, Bresee JS, Miller MA, Glass RI. Global illness and deaths caused by rotavirus disease in children. Emerg Infect Dis. 2003;9(5):565572. doi: 10.3201/eid0905.020562

3. John J, Sarkar R, Muliyil J, Bhandari N, Bhan MK, Kang G. Rotavirus gastroenteritis in India, 2011-2013: Revised estimates of disease burden and potential impact of vaccines. Vaccine. 2014, 32(Suppl 1):A5-A9. doi: 10.1016/j.vaccine.2014.03.004

4. Raju B, Parikh RP, Vetter VV, Kolhapure S. Epidemiology of rotavirus gastroenteritis and need of high rotavirus vaccine coverage with early completion of vaccination schedule for protection against rotavirus diarrhea in India: A narrative review. Indian J Public Health. 2019;63(3):243-250 doi: 10.4103/ijph.IJPH_307_18

5. Crawford SE, Ramani S, Tate JE, et al. Rotavirus infection. Nat Rev Dis Primers. 2017;3:17083. doi: 10.1038/nrdp.2017.83

6. World Health Organization. Rotavirus vaccines. Wkly Epidemiol Rec. 2007 Aug 10;82(32):285-95. English, French. PMID: 17691162. [Last accessed on 20 July 2020].

7. Arora R. Indian National Rotavirus Surveillance Network; Division of Epidemiology and Communicable Diseases; Indian Council of Medical Research 2014.

8. Shrivastava AK, Reddy NS, Giri S, et al. Burden and molecular epidemiology of Rotavirus causing diarrhea among under-five children: A hospital-based study from Eastern India. J Global Infect Dis. 2019;11(4):147152. doi: 10.4103/jgid.jgid_16_19

9. Centers for Disease Control and Prevention. Epidemiology and Prevention of Vaccine Preventable Diseases, Rotavirus; $13^{\text {th }}$ Edition 2015.

10. Gupta S, Singh KP, Jain A, Srivastava S, Kumar V, Singh M. Aetiology of childhood viral gastroenteritis in Lucknow, north India. Indian J Med Res. 2015;141(4):469-472. doi: 10.4103/0971-5916.159298

11. Burke RM, Tate JE, Kirkwood CD, Steele AD, Parashar UD. Current and new rotavirus vaccines. Curr Opin Infect Dis. 2019;32(5):435-444. doi: 10.1097/ QCO.0000000000000572

12. Kahn G, Fitzwater S, Tate J, et al. Epidemiology and prospects for prevention of rotavirus disease in India. Indian Pediatr. 2012;49(6):467-474. doi: 10.1007/ s13312-012-0076-7

13. Bhandari N, Rongsen-Chandola T, Bavdekar A, et al. Efficacy of a monovalent human-bovine (116E) rotavirus vaccine in Indian infants: a randomised double-blind placebo-controlled trial. Lancet. 2014;383(9935):2136-2143. doi: 10.1016/S01406736(13)62630-6

14. Giri S, Nair NP, Mathew A, et al. Rotavirus gastroenteritis in Indian children < 5 years hospitalized for diarrhoea, 2012 to 2016. BMC Public Health. 2019;19(1):69. doi: 10.1186/s12889-019-6406-0

15. Manual of rotavirus detection and characterization 
methods World Health Organization 2009. Department of Immunization, Vaccines and Biologicals $\mathrm{CH}-1211$ Geneva 27, Switzerland. [25 October 2018] https:// apps.who.int/iris/bitstream/handle/10665/70122/ WHO_IVB_08.17_eng.pdf?sequence=1\&isAllowed $=y$

16. World Health Organization Guideline: Updates on the management of severe acute malnutrition in infants and children, Geneva: World Health Organization; 2013. https://www.who.int/publications/i/ item/9789241506328

17. Ruuska T, Vesikari T. Rotavirus disease in Finnish children: use of numerical scores for clinical severity of diarrhoeal episodes. Scand J Infect Dis. 1990;22(3):259267. doi: $10.3109 / 00365549009027046$

18. John BM, Devgan A, Mitra B. Prevalence of rotavirus infection in children below two years presenting with diarrhoea. Medical Journal Armed Forces. 2014;70(2)116-119. doi: 10.1016/j.mjafi.2014.02.008

19. Banerjee I, Ramani S, Primrose B, et al. Comparative study of the epidemiology of rotavirus in children from a community-based birth cohort and a hospital in South India. J Clin Microbiol. 2006;44(7):2468-2474. doi: 10.1128/JCM.01882-05

20. Chavan NS, Tankhiwale N. Detection of human Rotavirus and Bacterial Enteropathogen in Acute Gastroenteritis in children below 5 years of age in Wardha. Int J Curr Microbiol App Sci. 2014;3(6):288291.

21. Rao P, Bele MK. Prevalence of rotavirus in acute pediatrics patients admitted to a tertiary care hospital in Mangalore. J Adv Sci Res. 2015:6(3):37-40.

22. Saravanan $P$, Ananthan $S$, Ananthasubramanian $M$. Rotavirus infection among infants and young children in Chennai, South India. Indian J Med Microbiol. 2004;22(4):212-221. doi: 10.1016/S02550857(21)02765-1

23. Dutta SR, Khalfan SA, Baig BH, Philipose L, Fulay Fil R. Epidemiology of rotavirus diarrhoea in children under five years in Bahrain. Int J Epidemiol. 1990;19(3):722727. doi: $10.1093 / \mathrm{ije} / 19.3 .722$

24. Nath G, Singh SP, Sanyal SC. Childhood diarrhoea due to rotavirus in a community. Indian J Med Res. 1992;95(4):259-262.

25. Sarangi R, Rath S, Dash M, Rath B, Lenka R K, Padhy $R N$. Prevalence of rotaviral diarrhoea in under-five hospitalized children in a tertiary care hospital of Eastern India. Egyptian Pediatric Association Gazette. 2015;63(2):46-51. doi: 10.1016/j.epag.2015.04.003

26. Kumar GCP, Giri S, Chawla-Sarkar M, et al. Epidemiology of rotavirus diarrhoea among children less than 5 years hospitalized with acute gastroenteritis prior to rotavirus vaccine introduction in India. Vaccine. 2020;38(51):8154-8160. doi: 10.1016/j. vaccine.2020.10.084

27. Nair NP, Reddy NS, Giri S, et al. Rotavirus vaccine impact assessment surveillance in India: protocol and methods. BMJ Open. 2019;9(4):e024840. doi: 10.1136/ bmjopen-2018-024840

28. Meel SK, Katewa $V$, Singh R, et al. The Burden of Rotavirus Gastroenteritis in Children: A Hospital-Based Prospective Study in Western Rajasthan. Cureus. 2020;12(10):e11020. doi: 10.7759/cureus.11020

29. Muendo C, Laving A, Kumar R, Osano B, Egondi T, Njuguna P. Prevalence of rotavirus infection among children with acute diarrhoea after rotavirus vaccine introduction in Kenya, a hospital cross-sectional study. BMC Pediatr. 2018;18(1):323. doi: 10.1186/s12887018-1291-8

30. Mathew MA, Paulose A, Chitralekha S, Nair MK, Kang G, Kilgore P. Prevalence of rotavirus diarrhea among hospitalized under-five children. Indian Pediatr. 2014;51(1):27-31. doi: 10.1007/s13312-014-0329-8

31. Babji S, Sindhu KN, Selvarajan S, et al. Persistence of G10P[11] neonatal rotavirus infections in southern India. J Clin Virol. 2021;144:104989. doi: 10.1016/j. jcv.2021.104989 